21 September 1999

JYFL-8/99

hep-ph/9909456

\title{
SCALING OF TRANSVERSE ENERGIES AND MULTIPLICITIES WITH ATOMIC NUMBER AND ENERGY IN ULTRARELATIVISTIC NUCLEAR COLLISIONS
}

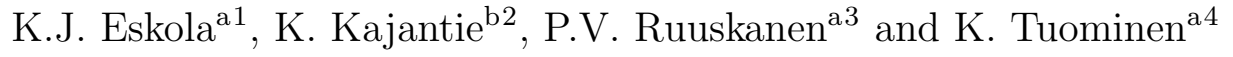 \\ a Department of Physics, P.O.Box 35, FIN-40351 Jyväskylä, Finland \\ b Department of Physics, P.O.Box 9, FIN-00014 University of Helsinki, Finland
}

\begin{abstract}
We compute how the initial energy density and produced gluon, quark and antiquark numbers scale with atomic number and beam energy in ultrarelativistic heavy ion collisions. The computation is based on the argument that the effect of all momentum scales can be estimated by performing the computation at one transverse momentum scale, the saturation momentum. The initial numbers are converted to final ones by assuming kinetic thermalisation and adiabatic expansion. The main emphasis of the study is at LHC and RHIC energies but it is observed that even at SPS energies this approach leads to results which are not unreasonable: what is usually described as a completely soft nonperturbative process can also be described in terms of gluons and quarks. The key element is the use of the saturation scale.
\end{abstract}

\footnotetext{
${ }^{1}$ kari.eskola@phys.jyu.fi

${ }^{2}$ keijo.kajantie@helsinki.fi

${ }^{3}$ vesa.ruuskanen@phys.jyu.fi

${ }^{4}$ kimmo.tuominen@phys.jyu.fi
} 


\section{Introduction}

In ultrarelativistic heavy ion collisions, the number of produced gluons and quarks with $p_{T}$ larger than some cut-off $p_{0}, N_{A A}\left(p_{0}, \sqrt{s}\right)$, increases [1]-[2] when $p_{0}$ decreases, when the size of the nucleus increases $\left(\sim d \sigma / \sigma_{\text {inel }} \sim A^{2} / R_{A}^{2} \sim A^{4 / 3}\right)$ and when $\sqrt{s}$ increases (since the small- $x$ enhancement [3] of the distribution functions becomes more effective). Shadowing will decrease the number [4], but NLO corrections [5] will increase it. At sufficiently large cut-off $p_{0} \gg \Lambda_{\mathrm{QCD}}$, the system of produced gluons and quarks is dilute and usual perturbation theory can be applied. However, at some transverse momentum scale $p_{0}=p_{\text {sat }}$ the gluon and quark phase space density saturates [7]-[8] and one does not expect further increase. In this case one may conjecture that the amount of $E_{T}$ produced at $p_{\text {sat }}$ gives a good estimate of the total $E_{T}$ produced in an average nucleus-nucleus collision: gluons with $p_{T} \gg p_{\text {sat }}$ carry lots of $E_{T}$ but are very rare, whereas gluons with $p_{T} \ll p_{\text {sat }}$ are numerous but carry little $E_{T}$. Doing the computation at $p_{\text {sat }}$ gives an estimate of the effect from all scales, both above and below $p_{\text {sat. }}$.

In this note, we shall work out the quantitative consequences of the above conjecture for the amount of $E_{T}$ initially produced in an $A+A$ collision at some large $\sqrt{s}$. The key element is the definition and computation of the saturation scale. For this, one first computes the number $N_{A A}\left(p_{0}, \sqrt{s}\right)$ of produced quanta including perturbatively all gluons and quarks with $p_{T}>p_{0}$. A saturation criterion is formulated and the solution of this gives $p_{\text {sat }}$. Evaluating $N_{A A}\left(p_{\text {sat }}, \sqrt{s}\right)$ and $E_{T}^{A A}\left(p_{\text {sat }}, \sqrt{s}\right)$ then gives the initial values. All quantities can well be fitted to a scaling law of the type $(\sqrt{s}$ is in units of $\mathrm{GeV}$ in the formulae)

$$
C A^{a}(\sqrt{s})^{b}
$$

possibly with an additional term constant in $s$.

This computation at $p_{\text {sat }}$ only gives initial values at the proper time $\tau_{i}=1 / p_{\text {sat }}$ and not yet experimentally measurable quantities. To get these, one has to trace the system through the entire set of expansion, hadronisation and decoupling stages. We shall also discuss this assuming kinetic thermalisation of the gluonic component and adiabatic expansion.

One is accustomed to considering the average events at SPS energies $(\sqrt{s} \lesssim 20 \mathrm{GeV})$ as essentially soft, stringlike. It is somewhat surprising that this entirely hard, perturbative, treatment will be seen to give numbers which are not unreasonable even at these relatively low energies. This phenomenon has also been pointed out in [9, 10] in the framework of a parton cascade model. From the point of view of our study the crucial factor is the use of the saturation scale as the scale of dominant processes.

There are clearly many refinements (or objections) one can suggest to this approach. The most obvious is that one should try to spread out the relevant momentum scales around $p_{\text {sat }}$ or, in time, around $\tau_{i}=1 / p_{\text {sat }}$. Further, there certainly will be some entropy production during the expansion stage - that due to chemical equilibration 
has been estimated in [11. We will discuss these briefly, but the key issue anyway is the use of $p_{\text {sat }}$ as the dominant scale.

If $p_{\text {sat }}$ is very large, well into the perturbative regime, there are transverse scales $p_{T} \ll p_{\text {sat }}$ which are perturbative but for which transverse phase space occupation numbers are $\gg 1$. A classical field description is then appropriate [12]-[20], in analogy with the computation of the rate of baryon number violating reactions in electroweak theory 21].

\section{Initial values}

The computation is technically a standard pQCD one and uses the formulas in Section 2 of [22]. The parton distribution functions are from [23] and nuclear effects to them are implemented using the EKS98-parametrization [4]. The corresponding NLO corrections to $E_{T}$ within the appropriate acceptance region $(|y|<0.5$, all azimuthal angles around the beam direction, and $p_{T}>p_{0}$ ) have been computed in [5], see also [6]. This is an infrared safe computation. For, say, $p_{0}=2 \mathrm{GeV}$ and choosing all the scales as $p_{T}$, one finds $K=1.7$ at $\sqrt{s}=5500 \mathrm{GeV}, K=2.3$ at $\sqrt{s}=200 \mathrm{GeV}$ and $K=1.9$ at $\sqrt{s}=20 \mathrm{GeV}$. In principle, the $K$-factor should be computed at the saturation scale, but in view of this small range of variation we shall take $K=2$. Anyway the NLO corrections to $N$ cannot be computed in an IR safe manner without introducing some additional regulator.

The calculation now proceeds as follows. First compute the average number $N_{k}\left(p_{0}\right)$, $k=g, q, \bar{q}$, of quanta produced in an average central $A+A$ collision within $|y|<0.5$ (for brevity, the fact that various quantities may depend on $A$, centrality, $\sqrt{s}$ or $p_{0}$ is not explicitly marked in what follows). The saturation limit is then obtained from the equation

$$
N\left(p_{0}\right)=\sum_{k=g, q, \bar{q}} N_{k}\left(p_{0}\right)=p_{0}^{2} R_{A}^{2},
$$

where $R_{A}=1.12 A^{1 / 3} \mathrm{fm}$. This expresses the fact that at saturation $N\left(p_{\text {sat }}\right)$ quanta each with transverse area $\pi / p_{\text {sat }}^{2}$ fill the whole nuclear transverse area $\pi R_{A}^{2}$. No numerical or group theory factors nor powers of $g_{s}$ are included; these are anyway $\mathrm{O}(1)$ unless one discusses a parametric weak coupling limit $g_{s} \rightarrow 0$. All parton flavours are included, though at $p_{0}=p_{\text {sat }}$ gluons clearly dominate even at lowest energies. The way Eq. (2) determines $p_{\text {sat }}$ for $A=208$ and $\sqrt{s}=17,200,5500 \mathrm{GeV}$ is shown in Fig. 11. The numerical values computed for various $A$ and $\sqrt{s}$ form a family of curves shown in Fig. 4 (normalised to give final charged multiplicity). They can be well fitted by (the points with $\sqrt{s}>200 \mathrm{GeV}$ are used in determining the parameters, though the fit agrees well also with the points computed at lower $\sqrt{s}$ )

$$
N_{i}=N\left(p_{0}=p_{\text {sat }}\right)=1.383 A^{0.922}(\sqrt{s})^{0.383},
$$




$$
\begin{aligned}
p_{\text {sat }} & =0.208 \mathrm{GeV} A^{0.128}(\sqrt{s})^{0.191}, \\
p_{\text {sat }} N_{i} & =0.288 \mathrm{GeV} A^{1.050}(\sqrt{s})^{0.574} .
\end{aligned}
$$

With given assumptions ( $K=2$, certain set of parton distributions, certain method of implementing shadowing, certain scale choices, etc) we require the accuracy of the fit to be within $1 \%$ at the fitted region and quote the parameters with corresponding accuracy. Clearly there is a larger error related to the underlying theoretical assumptions (e.g. the $K$-factor) and to the whole starting point: the use of $p_{\text {sat }}$ as the dominant scale.

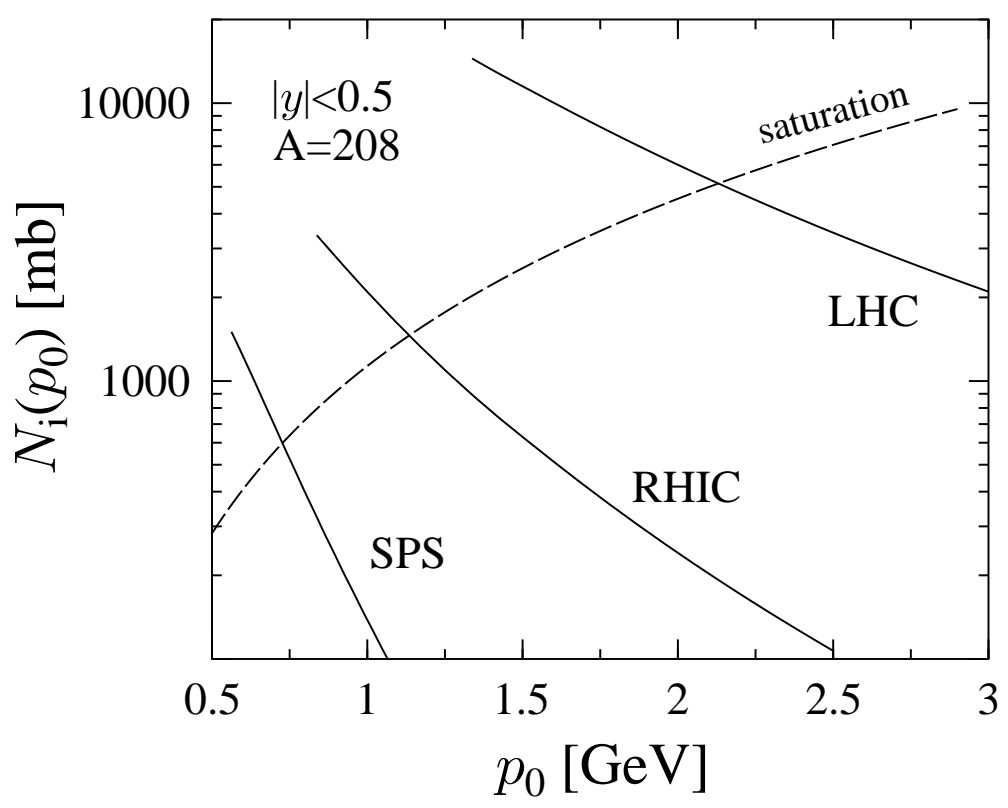

Figure 1: The average number of initially produced QCD-quanta with $p_{T} \geq p_{0}$ and $|y|<0.5$ as a function of the lower limit $p_{0}$ for central $\mathrm{Pb}-\mathrm{Pb}$ collisions at $\sqrt{s}=5500$ (LHC), 200 (RHIC) and $17 \mathrm{GeV}$ (SPS). The saturation scale $p_{\text {sat }}$ for $A=208$ is given by the points of intersection of the dashed curve "saturation" $\left(p_{0}^{2} R_{A}^{2}\right)$ with the curves $N\left(p_{0}\right)$.

Secondly, with the $p_{\text {sat }}$ so obtained, the initial values $E_{T i, k}$ and their sum $E_{T i}$ can be computed. The results for the sum are plotted in Fig. 2 and they behave as

$$
\begin{aligned}
E_{T i} & =0.386 \mathrm{GeV} A^{1.043}(\sqrt{s})^{0.595} \\
& =1.34 A^{-0.007}(\sqrt{s})^{0.021} p_{\text {sat }} N_{i} .
\end{aligned}
$$

By comparing (5) and (7) one observes that approximately $E_{T i} \approx$ const $p_{\text {sat }} N$. Actually the dependence on $\sqrt{s}$ and, in particular, on $A$ is so weak that one may question 
whether the deviation is a genuine physical effect. To interpret the result, assume first that precisely

$$
E_{T i}=C_{1} p_{\text {sat }} N_{i}
$$

with $C_{1}=$ constant. Then, using the initial volume estimate $V_{i}=\pi R_{A}^{2} / p_{\text {sat }}$ and the saturation relation $N_{i}=p_{\text {sat }}^{2} R_{A}^{2}$ one obtains

$$
\begin{aligned}
\epsilon_{i} & =\frac{E_{T i}}{V_{i}}=\frac{C_{1}}{\pi} p_{\text {sat }}^{4} \\
n_{i} & =\frac{N_{i}}{V_{i}}=\frac{1}{\pi} p_{\text {sat }}^{3} \\
\frac{\epsilon_{i}}{n_{i}} & =C_{1} p_{\text {sat }}
\end{aligned}
$$

Since the gluons are by far the dominant component, even at the SPS, let us convert $\epsilon_{i}$ to a temperature including only them, i.e., writing $\epsilon=16 \pi^{2} / 30 \cdot T^{4}$. Then

$$
T_{i}=\left(\frac{30 C_{1}}{16 \pi^{3}}\right)^{1 / 4} p_{\text {sat }} .
$$

Comparing (11) and (12), the boson gas thermal relation $\epsilon / n=2.70 T(=3.15 T$ for fermions) is seen to hold if $C_{1}^{3}=2.7^{4} 30 /\left(16 \pi^{3}\right), C_{1}=1.48$; very close to what the numerical calculation gives for it (see Eq. (7)).
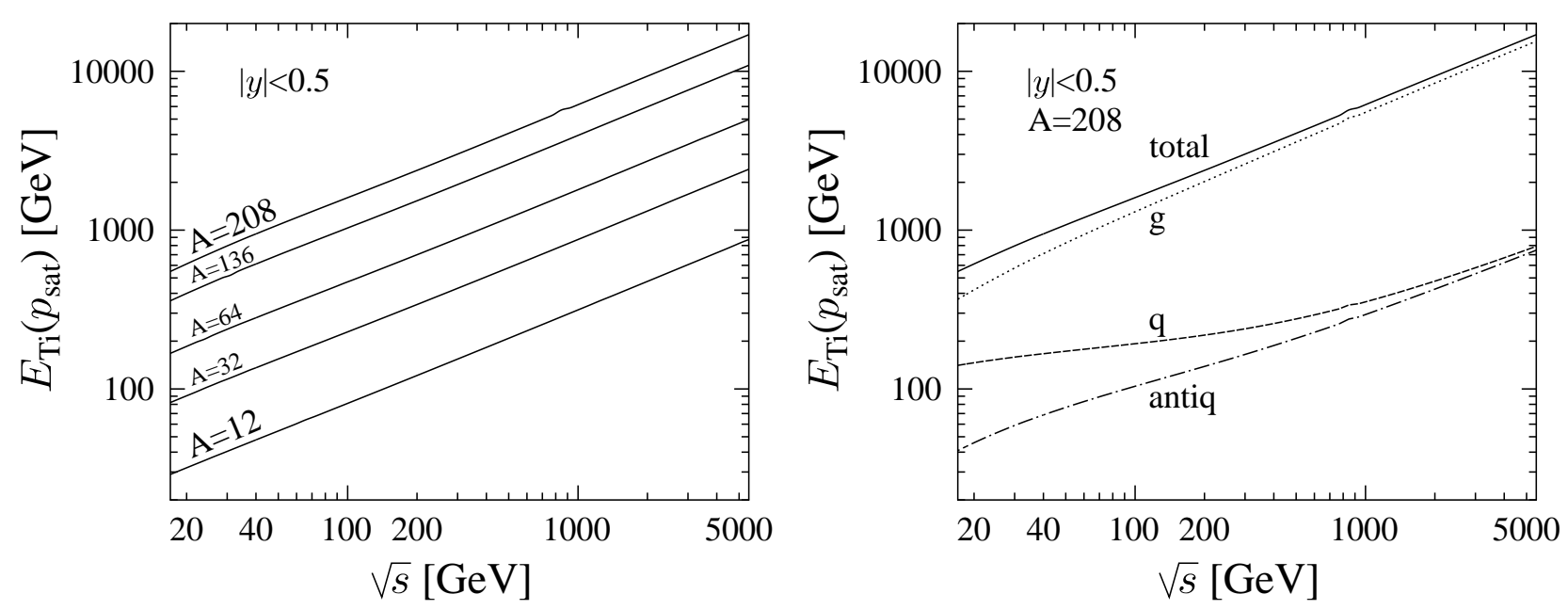

Figure 2: (a) The initial $E_{T}$ in $|y|<0.5$ in a central $A+A$ collision with $A=$ $12,32,64,136,208$ as a function of $\sqrt{s}$. (b) Decomposition of the initial $E_{T}$ into gluon, quark and antiquark components for $A=208$. 


\begin{tabular}{|c|c|c|c|c|c|c|}
\hline Quantity & $C$ & $a$ & $b$ & SPS & RHIC & LHC \\
\hline$N_{i}$ & 1.383 & 0.922 & 0.383 & 598 & 1440 & 5140 \\
$p_{\text {sat }} / \mathrm{GeV}$ & 0.208 & 0.128 & 0.191 & 0.73 & 1.13 & 2.13 \\
$E_{T i} / \mathrm{GeV}$ & 0.386 & 1.043 & 0.595 & 600 & 2360 & 17000 \\
$\epsilon_{i} /\left(\mathrm{GeV} / \mathrm{fm}^{3}\right)$ & 0.103 & 0.504 & 0.786 & 16.1 & 98.2 & 1330 \\
$n_{i} \cdot \mathrm{fm}^{3}$ & 0.370 & 0.383 & 0.574 & 16.0 & 59.8 & 401 \\
$T_{i} / \mathrm{GeV}$ & 0.111 & 0.126 & 0.197 & 0.39 & 0.62 & 1.19 \\
$E_{T c}$ & $3.477 T_{c}$ & 0.917 & 0.398 & 276 & 692 & 2600 \\
\hline
\end{tabular}

Table 1: Summary of the values of the scaling parameters $C, a, b$ in quantity $=C A^{a}(\sqrt{s})^{b}$, together with numerical values for $A=208$ at SPS, RHIC and LHC $(\sqrt{s}=20,200,5500$ $\mathrm{GeV}$ ). The numbers for $E_{T c}\left(\mathrm{Eq}\right.$. (24)) are for $T_{c}=0.18 \mathrm{GeV}$.

Including the small deviations from $E_{T i}=C_{1} p_{\text {sat }} N_{i}$ one finds, similarly:

$$
\begin{aligned}
\epsilon_{i} & =\frac{E_{T i}}{V_{i}}=0.103 \mathrm{GeVfm}^{-3} A^{0.504}(\sqrt{s})^{0.786}, \\
n_{i} & =\frac{N_{i}}{V_{i}}=0.370 \mathrm{fm}^{-3} A^{0.383}(\sqrt{s})^{0.574}, \\
T_{i} & =0.111 \mathrm{GeV} A^{0.126}(\sqrt{s})^{0.197}=0.534 A^{-0.002}(\sqrt{s})^{0.006} p_{\text {sat }}, \\
\frac{\epsilon_{i}}{n_{i}} & =2.52 A^{-0.005}(\sqrt{s})^{0.015} T_{i} .
\end{aligned}
$$

One observes that the ideal boson gas thermalisation relation $\epsilon / n=2.70 T$ holds almost independent of $A$ and $\sqrt{s}$ : the system satisfies this criterion of thermalisation right at production. Note that this applies to some degree even at the SPS. The scaling parameter values together with some relevant numerical values are summarised in Table 1.

It is also illustrative to consider the results for $g, q, \bar{q}$ separately. The $A$ and $\sqrt{s}$ dependences of the different components are almost the same but to account for the growing importance of the valence quark component towards smaller energies, one also has to include a constant term in the fit. For simplicity, we first make a four paramenter fit $A^{a}\left[C(\sqrt{s})^{b}+D\right]$ to the total initial multiplicity $N_{i}$ for $\sqrt{s}>40 \mathrm{GeV}$. With the powers $a$ and $b$ so fixed, we find $C$ and $D$ separately for each component. The result is

$$
\begin{aligned}
& N_{g}=A^{0.922}\left[1.065(\sqrt{s})^{0.404}-0.028\right], \\
& N_{q}=A^{0.922}\left[0.021(\sqrt{s})^{0.404}+0.778\right], \\
& N_{\bar{q}}=A^{0.922}\left[0.037(\sqrt{s})^{0.404}+0.287\right],
\end{aligned}
$$

and for the initial transverse energy

$$
E_{T g}=A^{1.044}\left[0.341(\sqrt{s})^{0.599}-0.398\right],
$$




$$
\begin{aligned}
& E_{T q}=A^{1.044}\left[0.0137(\sqrt{s})^{0.599}+0.494\right] \\
& E_{T \bar{q}}=A^{1.044}\left[0.0153(\sqrt{s})^{0.599}+0.153\right] .
\end{aligned}
$$

Note that the simple parametrisations above are only meant to reproduce the behaviour of the different components, not the small difference $N_{q}-N_{\bar{q}}$. They cannot be used to compute, e.g., the net quark number at the LHC, since $\left(N_{q}-N_{\bar{q}}\right) / N_{g}<$ the accuracy of the fits.

\section{Expansion stage}

We have now the initial values at $\tau_{i}=1 / p_{\text {sat }}$. To compare with experiment one has to follow the evolution of the system through expansion in the QCD plasma phase, phase transition, expansion in the hadronic phase and decoupling. Now that one has computed from perturbative QCD that the initial state very nearly satisfies the kinetic thermalisation condition $\epsilon / n=2.7 T$, there is some justification in assuming that also further expansion is locally thermal, i.e., entropy conserving. There is a large number of dissipative effects one can think of, but adiabatic boost invariant expansion is the baseline here.

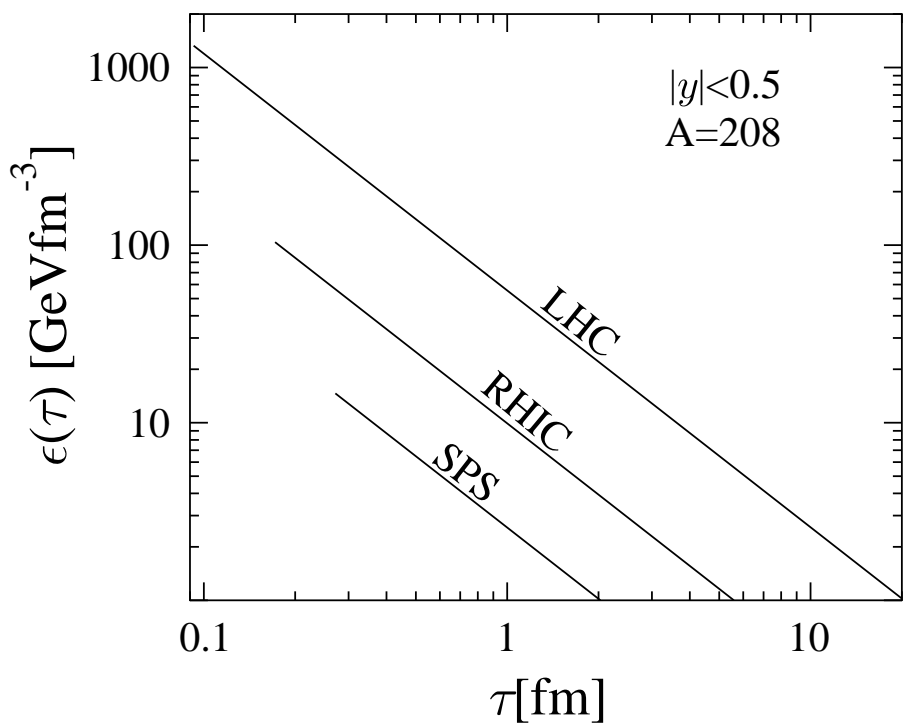

Figure 3: Proper time dependence of energy density during longitudinal expansion for LHC, RHIC and SPS with the initial values given in Table 1 .

Numerical examples of adiabatic expansion are shown in Fig. 3 for the initial onedimensional stage during which $\epsilon(\tau) \tau^{4 / 3}=$ constant. We start the expansion at the time 
$1 / p_{\text {sat }}$, although at lower energies this may be smaller than the transit time $2 R_{A} / \gamma=$ $4 R_{A} / \sqrt{s}$ of the nuclei. From the scaling formulas it actually follows that $2 R_{A} / \gamma<$ $1 / p_{\text {sat }}$ for

$$
\sqrt{s}>6.81 A^{0.570}(=143 \text { for } A=208) .
$$

We remind that the whole production process is effectively described as taking place at the time $1 / p_{\text {sat }}$.

Later on, at $\tau \sim \sqrt{3} R_{A}$, the system will enter a regime of longitudinal and transverse expansion, which has to be studied numerically [24]. In Fig. 3 we see that at the LHC the transverse expansion effects are likely to set in already in the plasma phase.

For the multiplicity the situation is simple: for an ideal system of bosons and fermions the total entropy is $S=3.60 N_{B}+4.20 N_{F}$ and this is constant per unit rapidity, as long as the expansion is boost invariant. (By definition, all quantities are always for $|y|<0.5$.) Thus, initially, $S_{i} \approx 3.6 N_{i}$. For the final hadronic gas $S_{i}=S_{f} \gtrsim 4 N_{f}$ so that $N_{f}=0.9 N_{i}$ : the number of final state hadrons is to up to $10 \%$ corrections equal to that of the initially produced partons (gluons) at the scale $p_{\text {sat }}$. The multiplicity prediction $N_{\mathrm{ch}}=2 / 3 * 0.9 N_{i}$ thus is directly given by Eq. (3) and is shown in Fig. 团.

Since NLO corrections to $E_{T}$ can be computed in an IR safe manner, one might prefer to compute the final entropy from the initial $E_{T}$ through the conversions $E_{T i} \rightarrow$ $\epsilon_{i} \rightarrow T_{i} \rightarrow S_{i}=S_{f}$. By making use of the fit (15), we obtain the following scaling law for the multiplicity of charged particles per unit rapidity:

$$
N_{c h}=\frac{2}{3} N_{f} \approx \frac{2}{3} \frac{S_{i}}{4}=\frac{2}{3} 1.16 A^{0.92}(\sqrt{s})^{0.40} .
$$

In view of the observed initial near thermalisation (Eq. (16)) this necessarily leads to the essentially same scaling in $A$ and $\sqrt{s}$ as that obtained from $N_{i}$ in Eq. (3). The comparison is shown in Fig. 因.

For $E_{T}$ the situation is more complicated. $E_{T}$ per unit rapidity decreases by the factor $T / T_{i}$ due to work done against expansion [24, 25]. On the other hand, the transverse expansion at later times will compensate for this effect.

To estimate the final $E_{T}$, we extract an approximate scaling law between the final average transverse momentum $\left\langle p_{T}\right\rangle$ and the final multiplicity $N_{f}$ from Fig. 7 of Ref. [24]:

$$
\left\langle p_{T}\right\rangle / \mathrm{GeV}=0.39+0.061 \ln \left(N_{f} / A\right) .
$$

By substituting our total multiplicity $N_{f}$, the final transverse energy can then be computed as

$$
E_{T f}=N_{f}\left\langle p_{T}\right\rangle .
$$

The transverse energies so obtained are shown in Fig. 5. Using Eq. (20) we get the scaling in $A$ and $\sqrt{s}$ :

$$
E_{T f}=0.46 A^{0.92}(\sqrt{s})^{0.40}[1-0.012 \ln A+0.061 \ln \sqrt{s}] .
$$




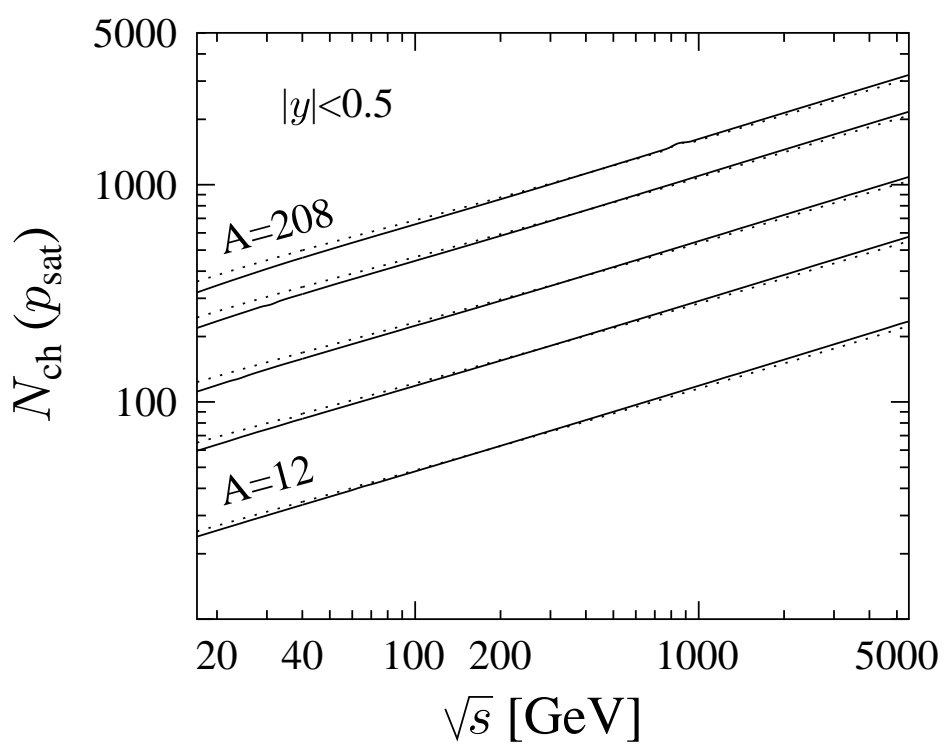

Figure 4: The number of charged particles per unit rapidity for $A=12,32,64,136,208$ as a function of $\sqrt{s}$, computed from Eq. (20) (solid lines) and compared with $2 / 3 * 0.9 N_{i}$ (dotted lines).

On the other hand, a simple estimate of the transverse energy $E_{T c}$ at the end of the plasma phase is obtained by neglecting the transverse expansion during the plasma phase. This gives

$$
\begin{aligned}
E_{T c}=\left(T_{c} / T_{i}\right) E_{T i} & =3.477 T_{c} A^{0.917}(\sqrt{s})^{0.398} \\
& =2.51 T_{c} A^{-0.005}(\sqrt{s})^{0.015} N_{i} .
\end{aligned}
$$

Using here $T_{c}=0.18 \mathrm{GeV}, E_{T c}$ approximates the final $E_{T}$ computed above very well. The comparison is shown in Fig. 5a.

Eq. (24) expresses a trivial fact: as long as the system is thermal, the energy per particle is $\sim 2.7 T$ - possibly even larger if also quarks enter chemical equilibrium. Evolution in the hadronic phase until decoupling will lead to a further reduction by the ratio $T_{\text {decoupling }} / T_{c} \lesssim 1$, but this is compensated for by the development of flow. Thus the net effect of the hadronic phase can be expected to be small, and $E_{T f} \approx E_{T c}$.

In Fig. 5b we show the difference between the initially produced $E_{T i}$ and the final, measurable, $E_{T f}$ for $A=208$. At the LHC the transverse energy drops by a factor $\sim 6$ and at RHIC by a factor $\sim 3.5$ due to $p d V$ work done against expansion. 

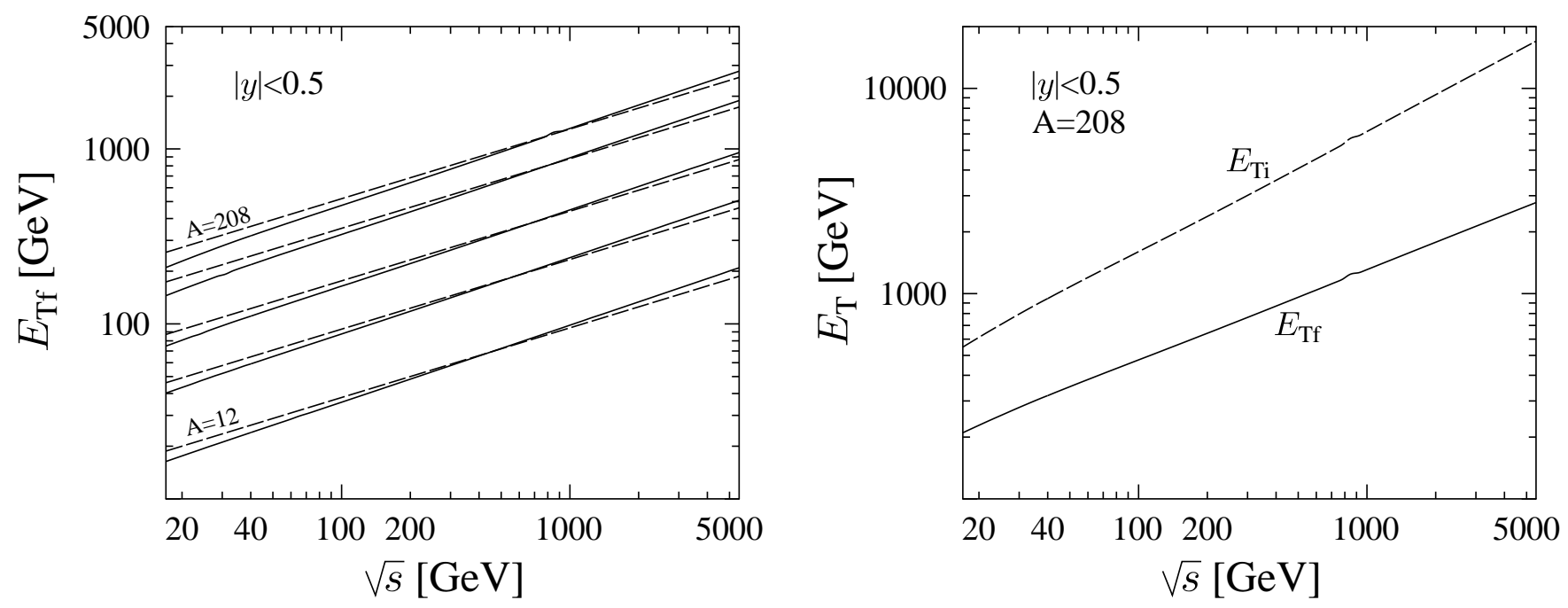

Figure 5: (a) The average final $E_{T}$ computed from Eq. (22) (solid lines) and from Eq. (24) with $T_{c}=0.18 \mathrm{GeV}$ (dashed lines) for $A=12,32,64,136,208$ as a function of $\sqrt{s}$. (b) Comparison of the initial and the final $E_{T}$ for $A=208$ as a function of $\sqrt{s}$.

\section{Discussion}

In the saturated scaling limit $\left(\sigma \sim p_{\text {sat }}^{-2}\right)$ one expects [1] the following $A$-scaling rules: First from Eq. (2) $), N \sim A^{4 / 3} / p_{\text {sat }}^{2}=\left(p_{\text {sat }} R_{A}\right)^{2}$, one obtains $p_{\text {sat }} \sim A^{1 / 6}$ and $N \sim$ $A$. Further, $E_{T i} \sim p_{\text {sat }} N \sim A^{7 / 6}$. Due to shadowing the observed $A$-exponents are somewhat less than these numbers, for $N_{i} 0.922$ instead of 1 and for $E_{T i} 1.043$ instead of 1.167. Anyway it is quite interesting to note these rather small exponents for this hard process. Note that for a fixed momentum scale without saturation the growth with $A$ is much more rapid: $N_{i} \sim E_{T i} \sim A^{4 / 3}$.

The growth with beam energy is rather rapid, the power of $\sqrt{s}$ is $b \approx 0.19$ for $p_{\text {sat }}$, $b \approx 0.38$ for $N_{i}$ and about the same for the final transverse energy, depending on the flow effects. A constant valence quark component can also be identified. The power of $\sqrt{s}$ depends on the phase space available and on the small- $x$ increase of the gluon distribution function.

The decrease of transverse energy in adiabatic expansion (Fig. 5b) is rather large, a factor $\sim 6(3.5)$ at the LHC (at RHIC). Provided that one can control flow effects numerically, a measurement of $E_{T f}$ will give further constraints on the degree of thermalisation in the system.

An interesting property of saturation calculations appears when considering them at 
SPS energies. Usually a heavy ion collision at the SPS, $\sqrt{s} \lesssim 20 \mathrm{GeV}$, is regarded as entirely soft, nonperturbative, dominated by stringlike effects. Here we have gone to the other extreme and have treated it in terms of the gluon and quark degrees of freedom at a very specific scale, $p_{T}=p_{\text {sat }}$. This scale is only $0.73 \mathrm{GeV}$ for $\mathrm{Pb}+\mathrm{Pb}$ collisions at the SPS, and perturbation theory at this scale, although $\alpha_{s}\left(p_{\text {sat }}\right) / \pi$ is clearly $<1$, is not expected to be very accurate (though it works well for, say, $\tau$ hadronic decays with $m_{\tau}=1.8 \mathrm{GeV}$ [26]). However, the numbers one obtains (Table 1) are not very far from the measured ones. The charged multiplicity per unit $y$ (actually $\eta$ ), is experimentally 27] about 400 for $\mathrm{Pb}+\mathrm{Pb}$ at $\sqrt{s}=17 \mathrm{GeV}$ while we obtain $N_{\mathrm{ch}}=330 \ldots 340$. For $\mathrm{S}+\mathrm{S}$ at $\sqrt{s}=20 \mathrm{GeV}$, the experimental value is $N_{\mathrm{ch}} \approx 50$ [28] while we get $N_{\mathrm{ch}} \approx 62 \ldots 64$, again not dramatically different. The average $E_{T}$ for $\mathrm{Pb}+\mathrm{Pb}$ is observed to be about $400 \mathrm{GeV}$ [29], somewhat larger than the numbers in Table 1. We have not in any way attempted to improve the quantitative agreement with present data. Maybe some kind of duality is at work here.

It is also notable that even at the SPS the system at $p_{\text {sat }}$ is dominantly gluonic: at $\sqrt{s}=17 \mathrm{GeV}$ the gluons take $65 \%$ of $E_{T i}$ and $73 \%$ of $N_{i}$. A related fact is that even at the SPS the thermalisation condition $\epsilon=2.7 T n$ is initially nearly satisfied.

The RHIC numbers $(A=208)$ are $\approx 1300$ for the total multiplicity $d N / d \eta$ (870 for $N_{\text {ch }}$ from (20)) and $\approx 660 \mathrm{GeV}$ for $d E_{T} / d \eta$ and will soon be measured. However, one can compare them with some historical numbers. In [i] the values $p_{\text {sat }}=0.95 \mathrm{GeV}$ and 1300 estimated for the multiplicity are very close to the ones computed here, but the discussion of $E_{T}$ included no $p d V$ work.

For the LHC, we get the charged particle multiplicity $d N_{c h} / d \eta \approx 3300$ and $d E_{T} / d \eta \approx$ $2900 \mathrm{GeV}$. These are some 25\% larger than the LHC-numbers in Ref. [22]. Now different, largely compensating, effects are included: shadowing and a slightly larger value of $p_{\text {sat }}$ decrease the numbers while $K=2$ instead of $K=1$ increases them.

Finally, some remarks are listed:

- The saturation criterion (2) has been presented here in a very simple form. Fundamentally, it is the result of a complicated dynamical computation containing numerical and group theory factors and powers of $g_{s}$ [8]. The precise value of all these will affect the final numbers. Taking the net effect to be $=1$ in (2) is a geometric estimate based on the uncertainty principle and there is no way of giving a controlled estimate of the error.

- The saturation criterion could also be formulated by applying it to the $p_{T^{-}}$ distribution, calculating it perturbatively for $p_{T}>p_{\text {sat }}$ and assuming that it is, say, constant for $p_{T}<p_{\text {sat }}$. This would lead to somewhat different numbers, but the error is like that under the previous point.

- To relate the present study to Ref. [30], it is perhaps interesting to compute the screening mass $m_{g}=g T$ at $T=T_{i}$. Using Eq. (15) we get $m_{g}=g_{s}\left(p_{\text {sat }}\right) T_{i}=$ 
$1.6 \ln ^{-1 / 2}\left(p_{\mathrm{sat}} / \Lambda_{\mathrm{QCD}}\right) A^{-0.002}(\sqrt{s})^{0.006} p_{\text {sat }}$. With the saturation scales in the region $p_{\text {sat }}=1 \ldots 2 \mathrm{GeV}$, we get $m_{g} \approx p_{\text {sat }}$, since $p_{\text {sat }} \approx 0.5 T_{i}$.

- The saturation criterion is formulated for quanta produced in the collision. The wave functions of the initial nuclei need not be saturated for the values of $A$ and $\sqrt{s}$ considered here.

- The starting point of these calculations of initial production of QCD quanta in ultrarelativistic central heavy ion collisions was that it is enough to consider one momentum scale, $p_{T}=p_{\text {sat }}$. The magnitude of the various quantities was then computed using methods appropriate for $p_{T} \geq p_{\text {sat }}$. One can, as well, attempt to use the range $p_{T} \leq p_{\text {sat }}$. This is a region of QCD in which genuinely new dynamical methods have to be developed. Numerical calculations also there are under way [18, 20]. However, whatever the method applied, it anyway applies only to the initial production $\left(1 / p_{\text {sat }}<\tau<1 \mathrm{fm}\right)$, leaving the treatment of the entire further evolution of the system open.

- There are many interesting and relevant questions concerning the subsequent flow, after initial production. The key issue is thermalisation, that of quarks, in particular [11, 31]. One could formulate the problem with all degrees of freedom and with some suppression factors (fugacities [31]) to account for the initial deficit of the quarks. To avoid this complication but to partly account for the quarks, we used the total energy density, including quarks, to find the initial temperature. The entropy increase associated with chemical equilibration of quarks was estimated to be about $15 \%$ at LHC in [11]. For SPS one has the problem of the long transit time.

\section{Conclusions}

We have in this article computed how the initial values of the energy and number densities of QCD matter produced in ultrarelativistic central heavy ion $(A+A)$ collisions depend on $A$ and $\sqrt{s}$. These values were converted to final observed numbers for $d N_{\mathrm{ch}} / d \eta$ and $d E_{T} / d \eta$ by assuming adiabatic expansion. This assumption is justified by the observation that the initial values very closely satisfy the kinetic thermalisation condition $\epsilon=2.7 n T$ for a dominantly gluonic system at all $A, \sqrt{s}$.

The computation is technically one in perturbative QCD, leading order with the NLO corrections estimated by a constant $K$-factor, and including nuclear shadowing, but also nonperturbative physics enters indirectly in that the computations are performed at one transverse momentum scale, the saturation momentum $p_{\text {sat }}$. This is determined by a geometric transverse saturation criterion and is supposed to effectively represent all scales, both those $>p_{\text {sat }}$ but also those $<p_{\text {sat }}$. In time, the initial production is 
assumed to effectively take place at $\tau=1 / p_{\text {sat }}$, instead of a gradual dumping of energy and entropy from the collision lasting until $\sim 1 / \Lambda_{\mathrm{QCD}}$.

Even at the SPS, where one normally expects soft stringlike physics to dominate, this entirely hard effective approach gives rather reasonable numbers. Maybe this gives some credibility to the predictions for RHIC and LHC, where one expects the approach to work better. From a purely theoretical point of view one can think of corrections from many different sources.

Acknowledgements: We thank L. McLerran and Al Mueller for discussions, B. Müller for extrapolating our curves to a region where we first dared not go and the Academy of Finland for financial support.

\section{References}

[1] J.P. Blaizot and A.H. Mueller, Nucl. Phys. B289 (1987) 847.

[2] K.J. Eskola, K. Kajantie and J. Lindfors, Nucl. Phys. B323 (1989) 37.

[3] N.H. Brook [for ZEUS \& H1 Collaborations], hep-ex/9805031.

[4] K.J. Eskola, V.J. Kolhinen and P.V. Ruuskanen, Nucl. Phys. B535 (1998) 351, hep-ph/9802350; K.J. Eskola, V.J. Kolhinen and C.A. Salgado, Eur. Phys. J. C9 (1999) 61, hep-ph/9807297.

[5] K.J. Eskola and K. Tuominen, in preparation.

[6] A. Leonidov and D. Ostrovsky, hep-ph/9811417.

[7] L.V. Gribov, E.M. Levin and M.G. Ryskin, Phys. Rept. 100 (1983) 1.

[8] A.H. Mueller and J. Qiu, Nucl. Phys. B268 (1986) 427.

[9] K. Geiger and D. K. Srivastava, Phys. Rev. C56 (1997) 2718, nucl-th/9706002.

[10] D. K. Srivastava and K. Geiger Phys. Lett. B422 (1998) 39, nucl-th/9708025.

[11] D.M. Elliott and D.H. Rischke, nucl-th/9908004.

[12] H. Ehtamo, J. Lindfors and L. McLerran, Z. Phys. C18 (1983) 341.

[13] L. McLerran and R. Venugopalan, Phys. Rev. D49 (1994) 2233, hep-ph/9309289.

[14] A. Kovner, L. McLerran and H. Weigert, Phys. Rev. D52 (1995) 6231, hepph/9502289.

[15] Y.V. Kovchegov and D.H. Rischke, Phys. Rev. C56 (1997) 1084, hep-ph/9704201. 
[16] M. Gyulassy and L. McLerran, Phys. Rev. C56 (1997) 2219, nucl-th/9704034.

[17] S.G. Matinian, B. Müller and D.H. Rischke, Phys. Rev. C56 (1997) 2191, nuclth/9705024.

[18] A. Krasnitz and R. Venugopalan, hep-ph/9809433.

[19] A.H. Mueller, hep-ph/9906322.

[20] A. Krasnitz and R. Venugopalan, hep-ph/9909203.

[21] G.D. Moore, hep-lat/9907009.

[22] K.J. Eskola and K. Kajantie, Z. Phys. C75 (1997) 515, nucl-th/9610015.

[23] M.Glück, E.Reya and A.Vogt, Z.Phys. C67 (1995) 433; H. Plothow-Besch, PDFLIB Version 7.09, W5051 PDFLIB, 1997.07.02, CERN-PPE.

[24] M. Kataja, P.V. Ruuskanen, L. McLerran and H. von Gersdorff, Phys. Rev. D34 (1986) 2755.

[25] M. Gyulassy, Y. Pang and B. Zhang, Nucl. Phys. A626 (1997) 999, nuclth/9709025.

[26] M. Girone and M. Neubert, Phys. Rev. Lett. 76 (1996) 3061, hep-ph/9511392.

[27] NA49 collaboration, H. Appelshauser et al., Phys. Rev. Lett. 82 (1999) 2471, nucl-ex/9810014.

[28] J. Bächler et al., Phys. Rev. Lett. 70 (1994) 1419.

[29] NA49 collaboration, S. Margetis et al., Phys. Rev. Lett. 75 (1995) 3814.

[30] K.J. Eskola, B. Müller and X.-N. Wang, Phys. Lett. B374 (1996) 20, hep$\mathrm{ph} / 9509285$.

[31] T.S. Biró, E. van Doorn, B. Müller, M.H. Thoma and X.-N. Wang, Phys. Rev. C48 (1993) 1275, nucl-th/9303004. 